\title{
Complete biodegradable nature of calcium hydroxylapatite after injection for malar enhancement: an MRI study
}

This article was published in the following Dove Press journal:

Clinical, Cosmetic and Investigational Dermatology

9 February 2015

Number of times this article has been viewed

\section{Tatjana Pavicic}

Private Practice, Munich, Germany
Correspondence: Tatjana Pavicic Privatpraxis, Maximilianstrasse 54, 80538 Munich, Germany

Tel +4989 I2II 4680

Email tatjana.pavicic@email.de
Background: Radiesse ${ }^{\sqrt{B}}$ (Calcium hydroxylapatite [CaHA]) is a biocompatible, injectable gel for facial soft tissue augmentation. It is a completely biodegradable filler and this is well documented, but objective imaging methods to confirm this property are scarce.

Methods: We present a case report in which CaHA was injected into the midface of a 50-year-old woman for volume restoration and shaping of the cheek region. On the right side of the face, $1.6 \mathrm{~mL}$ CaHA was injected as several (5-7) small depots $(0.1-0.2 \mathrm{~mL})$ using a $28 \mathrm{G} 3 / 4 \mathrm{inch}$ needle and the vertical supraperiosteal depot technique. On the contralateral side of the face, the subject received $1.6 \mathrm{~mL}$ CaHA over three entry points using a 27G 1 1/2 inch blunt cannula and the fanning technique. CaHA location and degradation were assessed using magnetic resonance imaging (MRI).

Results: CaHA appears as low-to-intermediate signal intensity on MRI images taken immediately after injection for malar enhancement with a symmetrical distribution. On MRI images taken 2.5 years after injection, no CaHA was visible but tissue volume remained increased, indicating a collagen-stimulating effect. The treatment was well tolerated.

Conclusion: In addition to producing long-lasting aesthetic and collagen-stimulating effects, MRI images confirm that CaHA is completely biodegradable with no product remaining 2.5 years after injection.

Keywords: aesthetic, biodegradable, calcium hydroxylapatite, MRI, Radiesse

\section{Introduction}

The longevity of dermal filler effects varies and is generally based on their composition and level of biodegradability within the tissues, although other factors such as the area treated, amount of product injected, depth of injection, age of the patient, their ability to synthesize new collagen, and their rate of metabolism, also play a role. ${ }^{1}$

Radiesse $^{\circledR}$ (Calcium hydroxylapatite [CaHA]; Merz Pharmaceuticals GmbH, Frankfurt, Germany) has a number of advantages as a dermal filler. It is biocompatible and does not require allergy testing prior to use. It is not derived from human or animal tissue, which minimizes the risk of contamination with pathogens. For a biodegradable filler, it is also long-lasting with an average duration of effect of around 15 months, and in some cases even longer than 30 months. ${ }^{2}$ As a result, fewer treatments are generally required to maintain results than for many other biodegradable fillers. ${ }^{3,4}$

The longevity of CaHA is a result of its twofold mechanism of action. The product is composed of synthetically produced smooth, uniform CaHA microspheres (diameter of 25-45 $\mu \mathrm{m}$ ) suspended in a sodium carboxymethylcellulose gel at a ratio of $30 \%$ microspheres to $70 \%$ gel by volume. In the first phase, the soluble 
carrier gel evenly distributes the CaHA microspheres at the injection site and provides an immediate volume correction. During the second phase, the gel gradually dissipates, ${ }^{5}$ leaving the CaHA microspheres at the injection site where they stimulate the production of endogenous collagen and dermal fibroblasts. ${ }^{6-8}$ Radiographic and computed tomographic studies of CaHA have shown that in many patients, the aesthetic corrections persist despite no CaHA being visible. ${ }^{9}$ Thus, the long-term correction provided by $\mathrm{CaHA}$ is not due to the continued presence of the microspheres, but to collagen production. In this case report, we use magnetic resonance imaging (MRI) to examine whether CaHA is completely biodegradable following injection for malar augmentation using two different injection techniques.

\section{Patient and methods}

The subject was a 50-year-old woman participating in a live injection session at an Expert Injectors meeting in Graz, Austria, in November $2010 .{ }^{10}$ She had a severity grade of 2 on the Merz scale for the midface ${ }^{11}$ and had received no prior treatments with CaHA or any other dermal filler. The woman was injected with CaHA supraperiostally on both sides of the face using two different techniques by a physician experienced in the use of CaHA. Volumes injected corresponded to the average usual volume of filler used in aesthetic practice. On the right side of the face she received $1.6 \mathrm{~mL}$ CaHA injected as several (5-7) small depots (0.1-0.2 mL) using a 28G 3/4 inch needle and the vertical supraperiosteal depot technique (VSDT) (Figure 1). ${ }^{12}$ The VSDT technique vertically deposits aliquots of filling agent at the periosteal level. The clinician gathers up the skin with the non-injecting hand for maximum penetration and pierces the skin at the thinnest entry point using a $90^{\circ}$ angle. At the level of the bone, the needle is repositioned and advanced slowly below the soft tissue before material deposition (Figure 2). Due to the presence of underlying bony support, little filling material is required for pronounced correction of the defect.

On the left side of the face, malar enhancement was achieved by injecting $1.6 \mathrm{~mL}$ CaHA over three entry points using a $27 \mathrm{G} 1$ 1/2 inch blunt cannula and the fanning technique, placing CaHA in the supraperiosteal plane (Figure 1). In the fanning technique, linear threading injections are made from a single insertion point in a fan-like pattern.

Post-treatment, both injection sites were gently massaged for optimal distribution of the injected material. Prefilled $1.5 \mathrm{~mL}$ syringes of CaHA were combined immediately pretreatment with $0.3 \mathrm{~mL}$ of lidocaine $2 \%$ and thoroughly mixed. The woman also received injection of a hyaluronic acid (0.2 mL Belotero Balance $\left.{ }^{\circledR}\right)$ using a $30 \mathrm{G} 1 / 2$ inch needle on both sides of the face in the tear trough region. For this treatment, a linear threading technique was used on the right side of the face and a VSDT technique on the left side of the face (Figure 1).

Two subjects were initially planned for this case study, but the second woman was unable to have repeat MRI because of planned surgery. The first MRI was performed with a Siemens Magnetom Essenza 1.5 Tesla and the second with a Siemens Magnetom Skyra 3.0 Tesla (Siemens Medical Solutions, Erlangen, Germany). Using T1- and T2-weighted Turbo Spin Echo sequences and fat suppression, axial, coronal, and
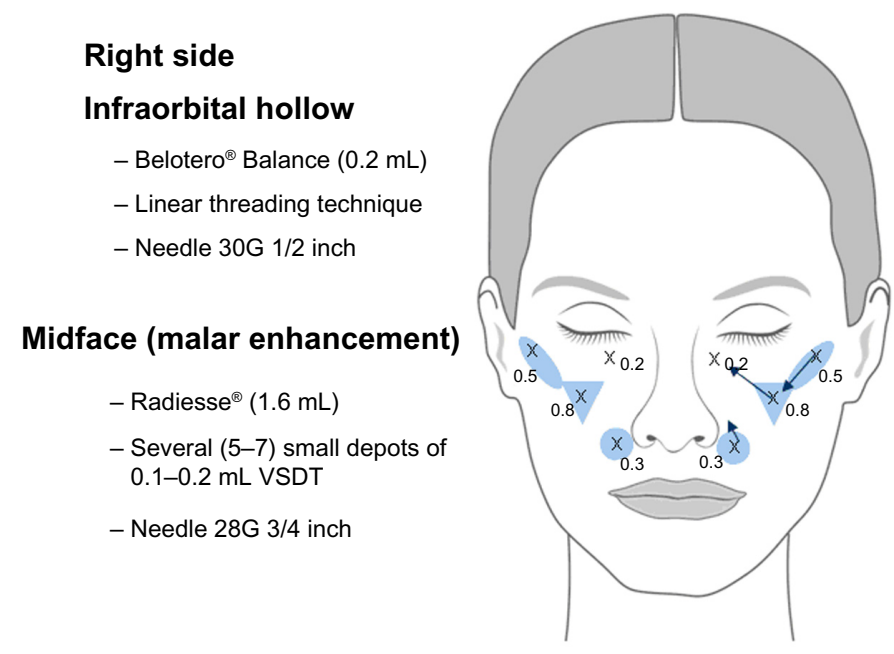

\section{Left side}

Infraorbital hollow

- Belotero ${ }^{\circledR}$ Balance $(0.2 \mathrm{~mL})$

- VSDT technique

- Needle 30G 1/2 inch

Midface (malar enhancement)

- Radiesse ${ }^{\circledR}(1.6 \mathrm{~mL})$

- Fanning technique

- Cannula 27G 1 1/2 inch

Dilution of Radiesse ${ }^{\circledR} 1.5 \mathrm{~mL}$ : $0.3 \mathrm{~mL}$ lidocaine $2 \%$

Figure I Injection scheme.

Notes: Crosses indicate insertion points. Arrows indicate direction of linear threads in fanning technique. Abbreviation: VSDT, vertical supraperiosteal depot technique. 

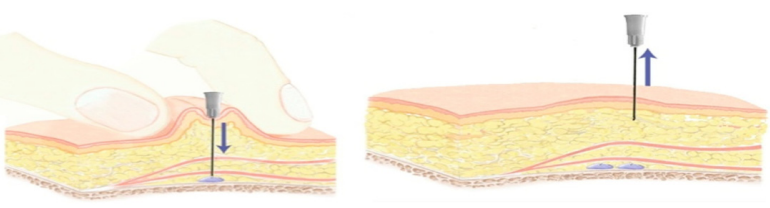

Figure 2 Illustration of the vertical supraperiosteal depot technique.

Notes: The clinician gathers up the skin with the non-injecting hand for maximum penetration and pierces the skin at the thinnest entry point using a $90^{\circ}$ angle. At the level of the bone, the needle is repositioned and advanced slowly below the soft tissue before material deposition.

sagittal series were obtained. Although two different MRI systems were used for the pre- and post-treatment assessment, the images were selected according to anatomical area and injection site so as to be comparable. The images differ only in their resolution, which was greater with the Magnetom Skyra 3.0 Tesla. The distribution of CaHA was evaluated on post-injection images taken 1 day after CaHA injection. The resorption of CaHA was quantified on MRI images acquired in April 2013, approximately 2.5 years after initial injection. Anatomical markers were used to allow accurate repositioning of the MRI images between successive acquisition procedures. MRI examinations were also assessed for collagen formation, formation of nodules, and signs of inflammation. Facial photographs were taken pre- and post-injection (Figure 3). Written informed consent was obtained.

\section{Results}

MRI images taken immediately after injection of CaHA for malar enhancement showed a symmetrical distribution of the filler in the supraperiosteal plane (Figure 4). Images taken approximately 2.5 years after injection revealed no presence of $\mathrm{CaHA}$, indicating that all the injected material had been reabsorbed and thus confirming the biodegradable nature of the product (Figure 5). As illustrated in Figure 5, MRI examination revealed no local soft tissue edema, no asymmetry, and no pathologically enlarged regional lymph nodes. MRI findings were unremarkable on both sides of the orbit, with no evidence of para- or retrobulbar infiltration or mass. No complications were reported during the 2.5 years since the injection took place.
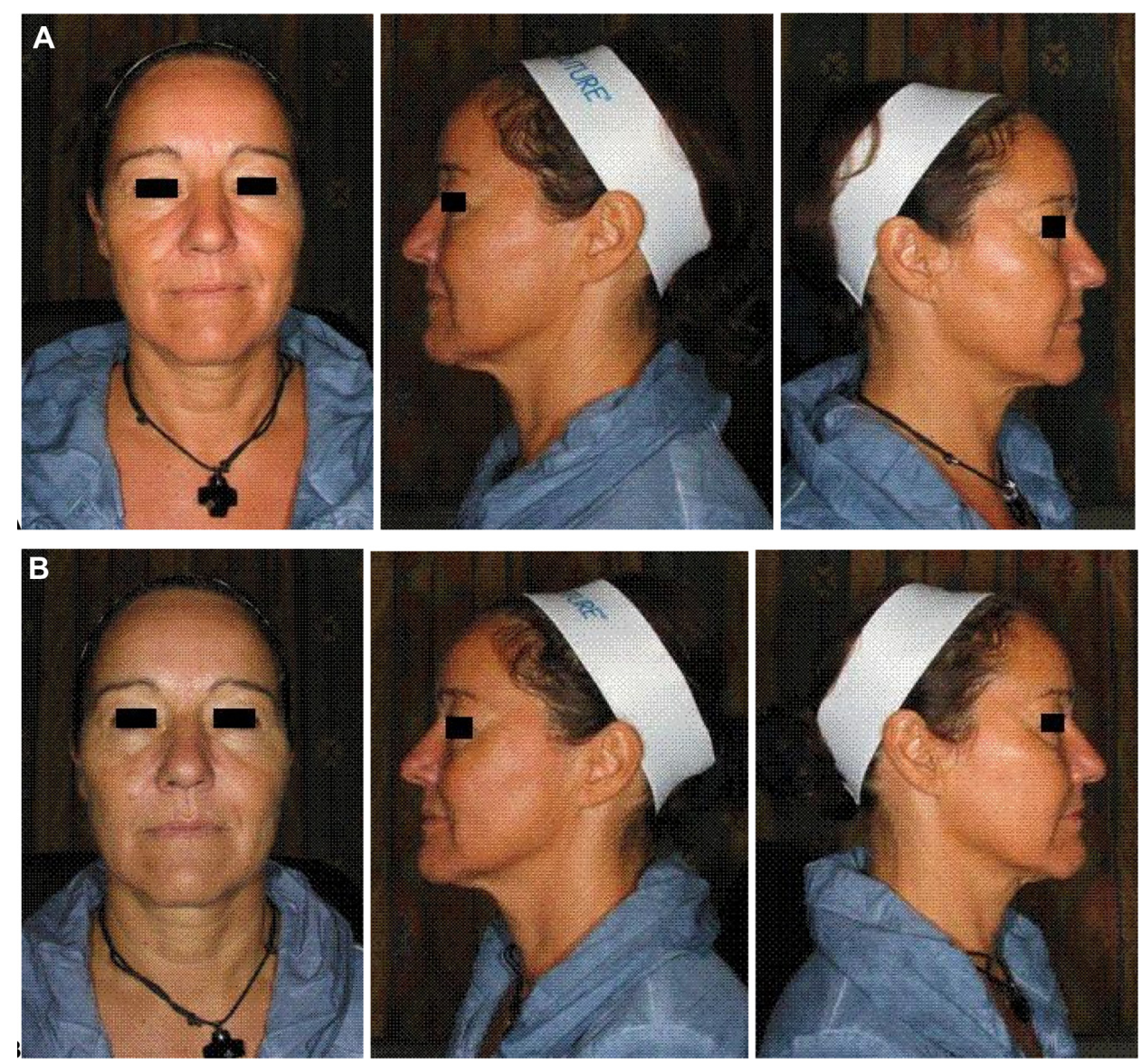

Figure 3 (A) Pre- and (B) immediate post-treatment photos after cheek augmentation and infraorbital hollow correction with I.6 mL CaHA (cheek) and $0.2 \mathrm{~mL}$ hyaluronic acid (infraorbital hollow).

Abbreviation: CaHA, Calcium hydroxylapatite. 

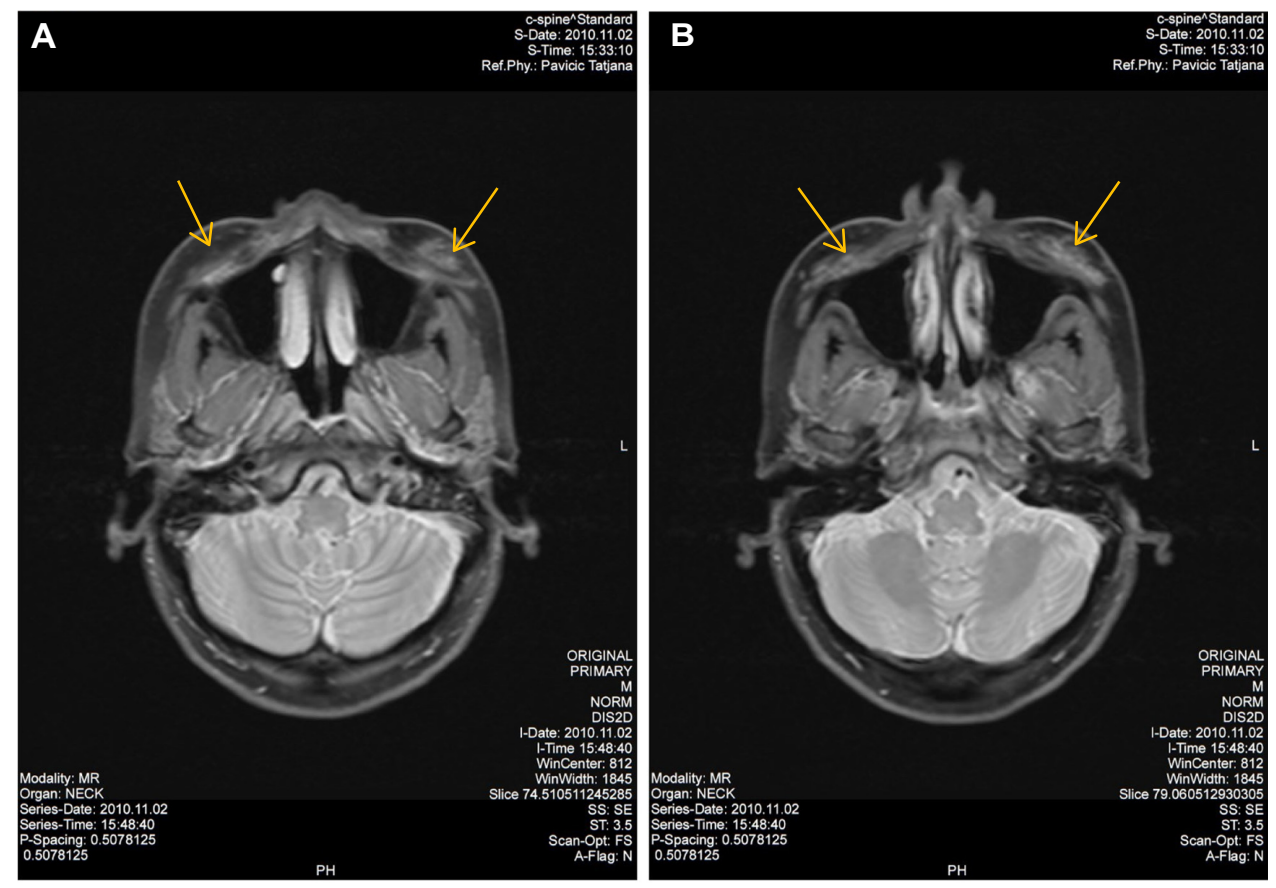

Figure 4 Horizontal sections from the cheek showing CaHA over a certain area corresponding the 3-dimensional cheek volumetry.

Notes: (A,B) MRI images acquired immediately after injection of CaHA for the cheek augmentation with a Siemens Magnetom Essenza I.5 Tesla. Product shown over two MRI sections.

Abbreviations: MRI, magnetic resonance imaging; CaHA, Calcium hydroxylapatite.

\section{Discussion}

Despite the popularity of dermal fillers, the physiological processes involved in the localization and subsequent resorption of injected products in skin tissues are poorly documented. High-resolution MRI offers an efficient noninvasive method for visualizing any dermal filler that has different MRI relaxation times from subcutaneous tissue. It can also be used for characterizing tissue changes, as well as for detecting nodules or indurations that may turn out to be foreign body granulomas on histological examination, fibrosis, and inflammation. The MRI images from this case report show that approximately 2.5 years after initial injection of CaHA no product is visible, confirming the biodegradable nature of CaHA. There were also no signs of (even hypothetical) bone formation after supraperiosteal injection of CaHA. The findings were the same on both sides of the face, and thus the resorption of CaHA was not influenced by the method of injection.

The results confirm previous radiographic and computed tomographic studies of CaHA, which show that in many patients the aesthetic corrections persist despite no CaHA being visible. ${ }^{9}$ A histological study has shown that the CaHA microspheres are resorbed by 12 months. ${ }^{13}$ The CaHA microspheres are gradually broken down into calcium and phosphate ions and eliminated through the body's physiological excretory processes. ${ }^{6}$ All of the components in the CaHA dermal filler are therefore fully metabolized over time. The end result is a highly biocompatible, long-lasting filler with similar characteristics to adjacent tissue.

Facial soft tissue undergoes significant deterioration over time, with the most dramatic changes between the ages of 30 and 60 years in the temporal, infraorbital, and lateral and medial cheek areas. ${ }^{14}$ Soft tissue augmentation and volume correction in these areas is an effective strategy for facial rejuvenation. CaHA achieves its aesthetic effect in a two-stage process. Immediate volume replacement is provided by the carrier gel instantly after injection. The biostimulatory CaHA particles then continue to provide volume correction and skin tightening even after the carrier gel has dissipated by stimulating connective and fat tissue to produce collagen, before the CaHA particles themselves are resorbed. This mechanism of action may partly explain why a smaller volume of CaHA tends to produce equal or greater effect and longer-lasting aesthetic results after single injection/administration than a greater volume of many comparable biodegradable fillers. ${ }^{3,4}$

Biodegradable fillers are generally preferred over permanent fillers. A major reason for this is safety, because if complications occur, they will generally disappear spontaneously. Biodegradable fillers also leave open the possibility of surgical correction in the future, whereas with permanent fillers, surgery may no longer be an option. The safety record of 

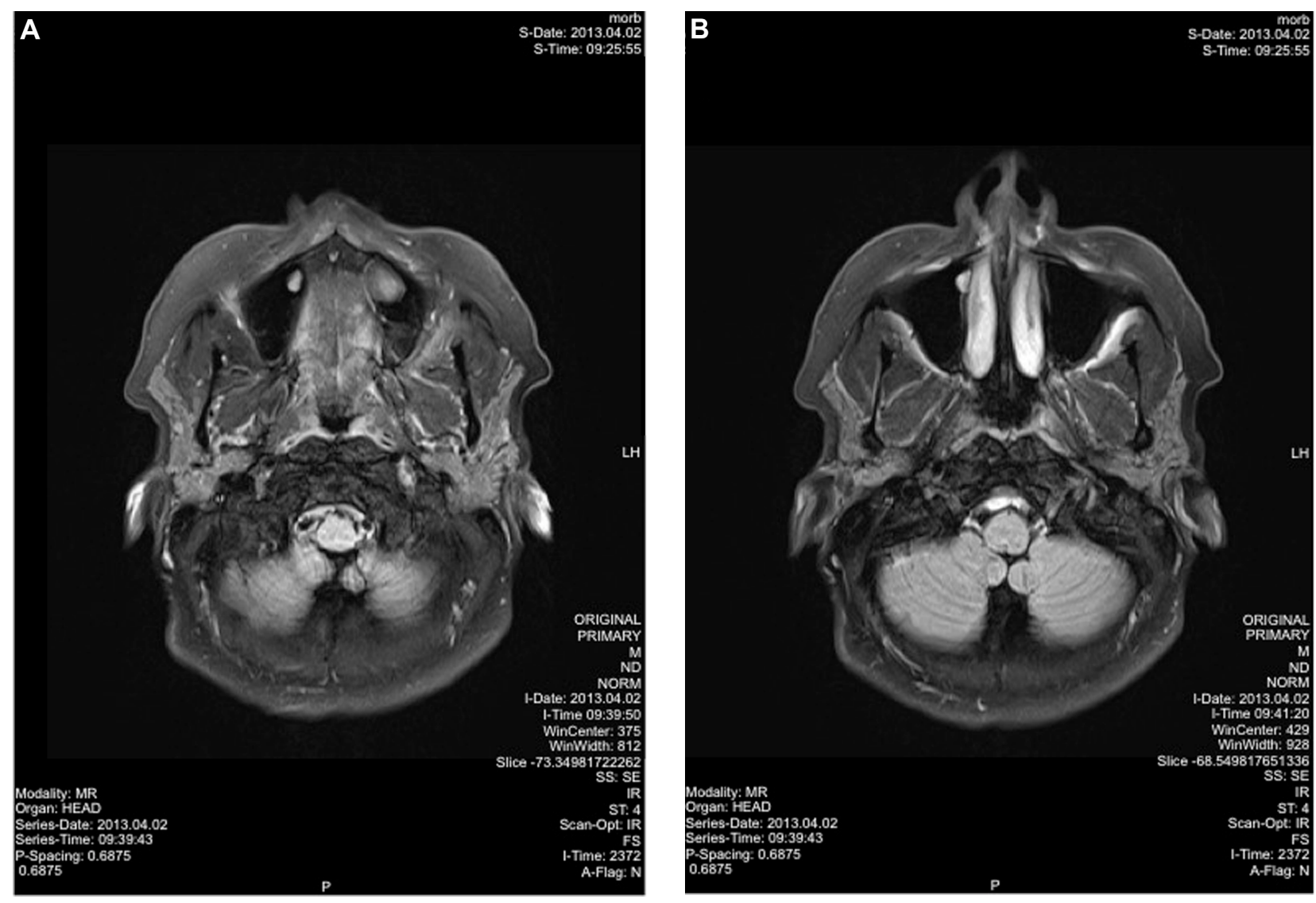

Figure $\mathbf{5}$ Horizontal sections from the corresponding cheek area without $\mathrm{CaHA}$.

Notes: (A,B) MRI images acquired approximately 2.5 years after $\mathrm{CaHa}$ injection with a Siemens Magnetom Skyra 3.0 Tesla. No CaHA was detectable in any of the corresponding MRI sections.

Abbreviations: MRI, magnetic resonance imaging; CaHA, Calcium hydroxylapatite.

CaHA is very well established and has been documented in both short- and long-term trials ${ }^{2,3,15-18}$ (reviewed by Pavicic ${ }^{19}$ ) and confirmed in over 160 scientific publications. The woman in the current case report experienced no adverse events over 2.5 years of follow-up.

Only a few studies in the literature have described the signal characteristics of CaHA on MRI. ${ }^{16,20-22}$ In these, CaHA appears as low-to-intermediate signal intensity on both T1- and T2-weighted sequences. ${ }^{20,22}$ The current case report indicates that $\mathrm{CaHA}$ is completely resorbed within 2.5 years after supraperiosteal injection for malar enhancement. Although the author is aware of no studies that have quantitatively measured the resorption of CaHA, computed tomographic scans following treatment for human immunodeficiency virus-associated facial lipoatrophy show only residual amounts 12 months after injection. ${ }^{9}$ However, the clinical aesthetic effect is much longer as the CaHA microspheres stimulate collagen neogenesis. A recent study, which performed punch biopsies 4 and 9 months after supraperiosteal injection of CaHA into the postauricular area, has shown that CaHA stimulates the production of collagen type III and type I in a two-step process whereby collagen type I gradually replaces collagen type III. ${ }^{23}$ This is consistent with the process of remodeling and collagen production that occurs under physiologic conditions, and contributes to an average duration of effect of around 15 months, and in some cases even longer than 30 months. ${ }^{2}$ CaHA therefore fills a clinical niche for those patients who wish to have a longerlasting filler, but one that is not permanent. Further studies are warranted to quantify the resorption of CaHA and how it dissipates over time. This would be useful for predicting the duration of CaHA and for comparing the longevity of CaHA with other biodegradable filler agents.

Rheological studies have shown that CaHA has high viscosity and elasticity, which prevent it from migrating from the injection site and resisting applied forces. ${ }^{24}$ To date, no MRI studies have quantified whether there is any lateral diffusion of CaHA. However, an electron microscope study of tissue samples taken post-injection in the postauricular area showed the CaHA particles beginning to dissolve, but 
no tissue migration, the product remaining well located in the dermal/subcutaneous junction. ${ }^{6} \mathrm{MRI}$ has been used to monitor the diffusion and progressive degradation of a hyaluronic acid (HA; Juvederm 3, Allergan Inc., Irvine, CA, USA) over time. ${ }^{25}$ The HA was found to diffuse at the dermis/hypodermis interface with a maximum extension at about 4 months. After this lateral diffusion, the HA product was progressively resorbed or degraded. ${ }^{25}$

\section{Conclusion}

CaHA is a valuable and effective injectable in the armamentarium of dermal filler options. It stimulates physiological collagen production and offers excellent aesthetic results with long-lasting effects for a variety of aesthetic indications, while remaining highly biocompatible with human tissue. MRI data from this case report also confirm that CaHA is completely biodegradable, with no product remaining 2.5 years after injection.

\section{Acknowledgments}

The author would like to acknowledge Dr Thomas Rappl, Senior Physician at the Department for Plastic, Aesthetic and Reconstructive Surgery, Medical University of Graz, Austria; and University Professor Dr Dieter Szolar, Diagnostikum Graz Süd West GmbH, Graz, Austria. This work was supported by Merz Pharmaceuticals. Jenny Grice provided help with medical writing.

\section{Disclosure}

Dr Pavicic is a speaker and consultant for Merz Pharmaceuticals and Galderma S.A., and reports no conflict of interest in this work.

\section{References}

1. Mahmoud BH, Ozog DM. Radiesse ${ }^{\circledR} /$ Radiesse $^{\circledR}$ with Lidocaine. In: Carruthers J, Carruthers A, editors. Soft Tissue Augmentation: Procedures in Cosmetic Dermatology Series. 3rd edition. Amsterdam: Elsevier; 2012:28-33.

2. Bass LS, Smith S, Busso M, McClaren M. Calcium hydroxylapatite (Radiesse) for treatment of nasolabial folds: long-term safety and efficacy results. Aesthet Surg J. 2010;30(2):235-238.

3. Moers-Carpi M, Vogt S, Santos BM, Planas J, Vallve SR, Howell DJ. A multicenter, randomized trial comparing calcium hydroxylapatite to two hyaluronic acids for treatment of nasolabial folds. Dermatol Surg. 2007;33(Suppl 2):S144-S151.

4. Moers-Carpi MM, Tufet JO. Calcium hydroxylapatite versus nonanimal stabilized hyaluronic acid for the correction of nasolabial folds: a 12-month, multicenter, prospective, randomized, controlled, split-face trial. Dermatol Surg. 2008;34(2):210-215.

5. Mayer R, Lightfoot M, Jung I. Preliminary evaluation of calcium hydroxylapatite as a transurethral bulking agent for stress urinary incontinence. Urology. 2001;57(3):434-438.
6. Marmur ES, Phelps R, Goldberg DJ. Clinical, histologic and electron microscopic findings after injection of a calcium hydroxylapatite filler. J Cosmet Laser Ther. 2004;694):223-226.

7. Berlin AL, Hussain M, Goldberg DJ. Calcium hydroxylapatite filler for facial rejuvenation: a histologic and immunohistochemical analysis. Dermatol Surg. 2008;34(Suppl 1):S64-S67.

8. Coleman KM, Voigts R, DeVore DP, Termin P, Coleman WP 3rd. Neocollagenesis after injection of calcium hydroxylapatite composition in a canine model. Dermatol Surg. 2008;34(Suppl 1):S53-S55.

9. Carruthers A, Liebeskind M, Carruthers J, Forster BB. Radiographic and computed tomographic studies of calcium hydroxylapatite for treatment of HIV-associated facial lipoatrophy and correction of nasolabial folds. Dermatol Surg. 2008;34(Suppl 1):S78-S84.

10. Carruthers J, Rzany B, Sattler G, Carruthers A. Anatomic guidelines for augmentation of the cheek and infraorbital hollow. Dermatol Surg. 2012;38(7 Pt 2):1223-1233.

11. Carruthers J, Flynn TC, Geister TL, Görtelmeyer R, et al. Validated assessment scales for the mid face. Dermatol Surg. 2012;38(2 Spec No):320-332.

12. Sattler G. The tower technique and vertical supraperiosteal depot technique: novel vertical injection techniques for volume-efficient subcutaneous tissue support and volumetric augmentation. $J$ Drugs Dermatol. 2012;11(8):S45-S47.

13. Lemperle G, Morhenn V, Charrier U. Human histology and persistence of various injectable filler substances for soft tissue augmentation. Aesthetic Plast Surg. 2003;27(5):354-366.

14. Wysong A, Joseph T, Kim D, Tang JY, Gladstone HB. Quantifying soft tissue loss in facial aging: a study in women using magnetic resonance imaging. Dermatol Surg. 2013;39(12):1895-1902.

15. Smith S, Busso M, McClaren M, Bass LS. A randomized, bilateral, prospective comparison of calcium hydroxylapatite microspheres versus human-based collagen for the correction of nasolabial folds. Dermatol Surg. 2007;33(Suppl 2):S112-S121.

16. Moers-Carpi M, Storck R, Howell DJ, Ogilvie P, Ogilvie A. Physician and patient satisfaction after use of calcium hydroxylapatite for cheek augmentation. Dermatol Surg. 2012;38(7 Pt 2):1217-1222.

17. Sadick NS. A 52 -week study of safety and efficacy of calcium hydroxylapatite for rejuvenation of the aging hand. J Drugs Dermatol. 2011;10(1):47-51.

18. Tzikas TL. A 52-month summary of results using calcium hydroxylapatite for facial soft tissue augmentation. Dermatol Surg. 2008;34(Suppl 1):S9-S15.

19. Pavicic T. Calcium hydroxylapatite filler: an overview of safety and tolerability. J Drugs Dermatol. 2013;12(9):996-1002.

20. Feeney JN, Fox JJ, Akhurst T. Radiological impact of the use of calcium hydroxylapatite dermal fillers. Clin Radiol. 2009;64(9):897-902.

21. van Rozelaar L, Kadouch JA, Duyndam DA, Nieuwkerk PT, Lutgendorff F, Karim RB. Semipermanent filler treatment of HIV-positive patients with facial lipoatrophy: long-term follow-up evaluating MR imaging and quality of life. Aesthet Surg J. 2014;34(1): 118-132.

22. Ginat DT, Schatz CJ. Imaging features of midface injectable fillers and associated complications. Am J Neuroradiol. 2013;34(8): $1488-1495$.

23. Yutskovskaya Y, Kogan E, Leshunov E. A randomized, split-face, histomorphologic study comparing a volumetric calcium hydroxylapatite and a hyaluronic acid-based dermal filler. J Drugs Dermatol. 2014;13(9): $1047-1052$.

24. Sundaram H, Voigts B, Beer K, Meland M. Comparison of the rheological properties of viscosity and elasticity in two categories of soft tissue fillers: calcium hydroxylapatite and hyaluronic acid. Dermatol Surg. 2010;36(Suppl 3):1859-1865.

25. Gensanne D, Josse G, Schmitt AM, Lagarde JM, Vincensini D. In vivo visualization of hyaluronic acid injection by high spatial resolution T2 parametric magnetic resonance images. Skin Res Technol. 2007;13(4): 385-389. 


\section{Publish your work in this journal}

Clinical, Cosmetic and Investigational Dermatology is an international, peer-reviewed, open access, online journal that focuses on the latest clinical and experimental research in all aspects of skin disease and cosmetic interventions. All areas of dermatology will be covered; contributions will be welcomed from all clinicians and basic science researchers globally. This journal is indexed on CAS. The manuscript management system is completely online and includes a very quick and fair peer-review system, which is all easy to use. Visit http://www.dovepress.com/testimonials.php to read real quotes from published authors.

Submit your manuscript here: http://www.dovepress.com/clinical-cosmetic-and-investigational-dermatology-journal 